\title{
Selective sphingosine 1-phosphate receptor 1 modulation augments thrombolysis of low-dose tissue plasminogen activator following cerebrovascular thrombosis
}

\section{Handong Li}

Tianjin Medical University General Hospital

\section{Ying Kong}

Tianjin Medical University General Hospital

\section{Wenyan Zhang}

Tianjin Medical University General Hospital

\section{Caiyun Qi}

Tianjin Medical University General Hospital

\section{Dan Wang}

Tianjin Medical University General Hospital

Qiang Liu ( $\sim$ qliu@tmu.edu.cn )

Tianjin Neurological Institute

\section{Research}

Keywords: 2-photon microscopy, tPA, thrombolysis, microcirculation, acute ischemic stroke

Posted Date: August 5th, 2021

DOI: https://doi.org/10.21203/rs.3.rs-764279/v1

License: (c) (i) This work is licensed under a Creative Commons Attribution 4.0 International License.

Read Full License 


\section{Abstract}

\section{Background}

Results from our recent study demonstrate that sphingosine-1-phosphate receptor 1 (S1PR1) modulation improves microvascular hemodynamics after cerebrovascular thrombosis. This study was to determine the microvascular hemodynamic effects of sub-thrombolytic dose of tPA combined with a selective S1PR1 modulator ozanimod in a mouse model of cerebrovascular thrombosis.

\section{Methods}

Microvascular circulation in mice was monitored in vivo by two-photon microscopy. Thrombosis was induced in cortical arterioles by laser irradiation. Arteriolar flow velocity was measured by line-scanning following plasma-labeling with fluorescein-dextran.

Results

Laser-induced thrombosis led to a persistent reduction of flow velocity in cortical arterioles. Subthrombolytic dose of tPA along with vehicle control did not improve the flow velocity in cortical arterioles following thrombosis. In contrast, sub-thrombolytic dose of tPA along with ozanimod dramatically restored flow velocity in cortical arterioles following thrombosis. Ozanimod did not affect coagulation and bleeding time. Notably, ozanimod reduced thrombus volume without altering microvascular lumen diameter. In addition, ozanimod reduced leukocyte components within the thrombus.

Conclutions

These findings demonstrate that the thrombolytic effect of sub-thrombolytic dose of tPA is markedly enhanced by S1PR1 modulation, implying that S1PR1 modulation may improve the therapeutic benefit of low-dose tPA in patients with acute ischemic stroke.

\section{Introduction}

Thrombolysis with tissue-type plasminogen activator (tPA) is the only FDA-approved pharmacological treatment of acute ischemic stroke (AIS), despite the increased risk of intracerebral hemorrhage. Although low-dose tPA thrombolysis reduces the risk of hemorrhage, the mortality and disability rates are increased in AIS patients receiving lower dose of tPA[1-4], presenting an unmet medical need for adjunctive therapies that can enhance the thrombolytic effects of low-dose tPA and maintain its safety advantage.

Sphingosine-1-phosphate receptor (S1PR) modulation provides protection in preclinical and clinical studies for ischemic stroke[5-7]. Fingolimod is a S1PR modulator that inhibits the transmigration of lymphocytes from peripheral lymphoid organs into neurovascular unit, and thereby reduces circulating lymphocytes within cerebrovascular compartment, leading to improved collateral flow and barrier integrity $[8,9]$. Evidence has demonstrated the efficacy of selectively targeting S1PR1 to attenuate 
ischemic brain injury, indicating that the benefit of fingolimod mainly results from its action on S1PR1[10-12]. In a recent study using two-photon laser-scanning microscopy in vivo, we reported that selective S1PR1 modulation improves microvascular circulation after cerebrovascular thrombosis[13], suggesting that efficacy of thrombolytic therapy in AIS may be enhanced by S1PR1 modulation. Therefore, this study explored the impact of a selective S1PR1 modulator ozanimod combined with subthrombolytic dose of tPA on microvascular hemodynamics using a mouse model of laser-induced cerebrovascular thrombosis.

\section{Material And Methods}

\section{Mice}

C57BL/6 mice, 8-10 weeks old (18-20g), were purchased from SPF Biotechnology Co., Ltd. (Beijing, China). Animals were housed under standardized light-dark cycle and provided with free access to food and water. QuickCalcs (GraphPad Software, La Jolla, CA, USA) was used to randomly assign animals into each treatment group. The coagulation time and bleeding time were measured in the same cohort of mice subjected to the measurement of flow velocity. All procedures were performed in accordance with the National Institutes of Health Guide for the Care and Use of Laboratory Animals and were approved by Animal Care and Use Committees of Tianjin Neurological Institute (Tianjin, China).

\section{Animal preparation for in vivo imaging}

Surgical preparation for in vivo imaging was performed as previously described[13, 14]. Briefly, mice were initially anesthetized by $4.5 \%$ isoflurane inhalation which was then maintained at $1-1.5 \%$ for the remainder of the study. Animals were placed in a stereotactic frame. Animal heads were immobilized and body temperature was recorded and maintained at a $37^{\circ} \mathrm{Cusing}$ circulating warm-water blanket. A midline scalp incision was made, and the periosteum was removed from the skull, exposing bone suture markings. Hemostasis was established in the soft tissues with monopolar cautery and in the bone by Gelfoam strips. A cranial window (diameter $6 \mathrm{~mm}$ ) was created over the right and left frontoparietal cortex with the use of a drill. The skull and dura were removed and covered with a removable cover glass (diameter $8.0 \mathrm{~mm}$ ) that was glued to the skull using dental acrylic cement to cover the craniotomy window. The space between the exposed brain surface and the cover glass was filled with PBS.

\section{2-photon laser scanning microscopy}

A customized microscope attached to a laser generator with 680- to1020-nm tuning range (Model: Chameleon coherent, UK), a multiphoton laser scanning microscope (FVMPE-RS, Olympus, USA) operated by FluoView software (FV31S, Olympus, USA), and a 25X water-immersion objective lens (NA1.05, Olympus, USA) were used for in vivo imaging. Texas Red-dextran (MW: $70 \mathrm{kDa}, 0.1 \mathrm{~mL}$ of $1 \%$ in saline; Invitrogen, USA) via the tail vein was used to visualize the vasculature and detect the motion of red blood cells (RBCs) using two-photon microscopy[15]. In each animal, cortical arterioles (diameter: 30-50 $\mathrm{mm}$, subsurface depth: 10-20 $\mu \mathrm{m}$ ) were imaged via z-scans and flow velocity was determined by repetitive 
line-scans performed along the central longitudinal axis of the selected vessels[16]. Linear shadows as a result of nonfluorescent particles within the vessel lumen was used to compute RBC velocity, which was proportional to the slope $\Delta \mathrm{x} / \Delta \mathrm{t}$.

Localized vascular injury was produced by laser irradiation as previously described[14]. Arteriolar injury was induced by delivering multiple pulse laser-irradiation on the arteriole $(\sim 30 \times 50 \mu \mathrm{m}$ ellipse field) for $\sim$ 6 minutes in a wavelength of $920 \mathrm{~nm}$ until thrombus formed and occupied the entire luminal crosssection. The onset of thrombosis was identified by the dilatation of the irradiated segment, the emergence of bright fluorescence along the vessel wall, and by a nonfluorescent mass within the irradiated arteriole. The laser pulse energy which ranges between 0.1 to $0.6 \mathrm{~mW}$ weak enough to avoid dye extravasation and vessel rupture. If there was occurrence of vascular rupture, the animal was not used.

Thrombus volume was measured by 3D image using two-photon microscope. Whole thrombus was scanned every $1 \mu \mathrm{m}$ from top to bottom under two-photon microscope at $60 \mathrm{~min}$ after thrombosis and up to $90 \mathrm{~min}$ after vehicle or Ozanimod treatment. Thrombus volume were determined by measuring the area of thrombus in a series of scanned sections, and then multiplying the section thickness $(1 \mu \mathrm{m})$.

\section{Drug administration}

Animals with successful arteriolar thrombosis were randomly treated with tPA, ozanimod or vehicle by intravenous tail vein injection. Ozanimod (MCE, Monmouth, $\mathrm{NJ}$ ) was dissolved in 5\% DMSO $+0.9 \%$ saline and stored at $4^{\circ} \mathrm{C}$ as we previously described[17]. Mice were treated with Vehicle $(100 \mu \mathrm{l})$, Vehicle $(50 \mu \mathrm{l})+$ low dose tPA $(5 \mathrm{mg} / \mathrm{kg}, 50 \mu \mathrm{l})$ or Ozanimod $(0.6 \mathrm{mg} / \mathrm{kg}, 50 \mu \mathrm{l})+$ low dose tPA $(5 \mathrm{mg} / \mathrm{kg}, 50 \mu \mathrm{l})$ at $60 \mathrm{~min}$ after vascular injury induced by laser irradiation.

\section{Assessments of bleeding time, coagulation time}

Mouse tails were transected at $2 \mathrm{~mm}$ from the tip with a scalpel blade and immersed in a $15 \mathrm{ml}$ clear tube with normal saline at $37^{\circ} \mathrm{C}$. Bleeding time was determined as time to cessation of bleeding within a 20 min observation period[15]. Bleeding was considered stopped if no bleeding was observed for $30 \mathrm{~s}$. For the coagulation time assessment, blood was drawn from the angular vein into a $1 \mathrm{~mm}$ diameter glass capillary after anesthesia. The capillary tube was filled completely full of blood, then laid flat on the bench top. Next, a small section of the end of the capillary tube was carefully snapped every $30 \mathrm{~s}$ until a fibrin thread appeared. Both bleeding time and coagulation time were measured at $90 \mathrm{~min}$ after treatment with Ozanimod $(0.6 \mathrm{mg} / \mathrm{kg}$, i.v.) or vehicle.

\section{Immunostaining}

After two-photon imaging, mice were sacrificed and perfused with $10 \mathrm{ml}$ cold PBS, followed by $10 \mathrm{ml} 4 \%$ paraformaldehyde perfusion solution. Brain tissues were harvested and fixed in $4 \%$ paraformaldehyde overnight, and then dehydrated with $30 \%$ sucrose. Frozen brain tissues were sectioned ( $20 \mu \mathrm{m}$ thickness) and incubated for $30 \mathrm{~min}$ at room temperature in blocking buffer (5\% goat serum, $1 \%$ BSA, $0.3 \%$ Triton X100). Brain sections were then incubated with primary antibodies against mouse CD45 (1:200, 13917 Cell 
Signaling, Danvers, MA) or CD3 $\left(10 \mu \mathrm{g} / \mathrm{mL}, \mathrm{MAB} 4841 \mathrm{R} \& \mathrm{D}\right.$, Minneapolis, MN) at $4^{\circ} \mathrm{C}$ overnight. After washing in PBS, brain sections were incubated with appropriate fluorochrome-conjugated secondary antibodies at $4^{\circ} \mathrm{C}$ overnight. DAPI (H-1200, Vector, Burlingame, CA) was used to counterstain cell nuclei. Confocal Z stacks were acquired from thrombus area labeled by Texas-Red-Dextran using a Zeiss 710 microscope with $\times 20$ objective, NA 0.8 (Oberkochen, Germany). Data were analyzed with Image J software (National Institutes of Health, Washington, DC).

\section{Statistical analysis}

Randomization was performed using GraphPad QuickCalcs. Measurements and data analysis were performed by investigators blinded to experimental treatments. Statistical significance was determined by two-tailed unpaired Student's t-test for two groups, one-way analysis of variance (ANOVA) followed by Tukey post-hoc test for multiple groups, or two-way ANOVA accompanied by Bonferroni post hoc test for multiple comparisons. Statistical analysis was performed using Prism 8.0 software (GraphPad, San Diego, CA). Data are shown as mean \pm SD. Values of $p<0.05$ were considered significant.

\section{Results}

\section{Effects of subthrombolytic dose of tPA on microvascular hemodynamics after thrombosis}

Laser irradiation was used to produce thrombosis in selected cortical arterioles (diameter: $30-50 \mu \mathrm{m}$ ) under 2-photon microscopy (Fig. 1A). Laser irradiation-induced thrombus occupies the luminal space without vessel rupture or subsequent dye extravasation (Fig. 1B). After thrombosis, flow velocity in selected cortical arterioles immediately declined and the volume of thrombus remained stable (Fig. 1B-C). The reduction of flow velocity persisted and was not altered by vehicle treatment (Fig. 1D).

After treatment at 60 min of thrombosis, mice received a subthrombolytic dose of tPA ( $5 \mathrm{mg} / \mathrm{kg}$, i.v. injection) along with vehicle. The volume of individual thrombus and flow velocity were monitored until 90 min after treatment (Fig. 2A). We did not find obvious changes of thrombus volume or restoration of flow velocity following administration of low-dose tPA ( $5 \mathrm{mg} / \mathrm{kg}$ ) along with vehicle (Fig. 2B-C).

\section{Selective S1PR1 modulation enhances thrombolytic effect of low-dose tPA}

To examine the impact of S1PR1 modulation thrombolytic effects of low-dose tPA, we monitored thrombus volume and microvascular flow velocity in mice receiving a selective S1PR1 modulator ozanimod $(0.6 \mathrm{mg} / \mathrm{kg}$, i.v.) along with low-dose tPA ( $5 \mathrm{mg} / \mathrm{kg}$, i.v.). We found a notable reduction of thrombus volume and a dramatic increase of flow velocity in mice receiving ozanimod along with lowdose tPA (Fig. 3A-C), suggesting that S1PR1 modulation enhances thrombolytic effect of low-dose tPA. 


\section{Selective S1PR1 modulation does not affect bleeding time and coagulation time}

To test whether S1PR1 modulation improves thrombolytic effect of low-dose tPA by its action on coagulation system, we measured bleeding time and coagulation time in mice receiving ozanimod or vehicle. We did not find discernable impact of ozanimod on coagulation time and bleeding time (Fig. 4AB), suggesting that the impact of ozanimod on the coagulation system is minimal.

\section{Selective S1PR1 modulation reduces thrombus volume and lymphocytes within thrombus.}

To understand how ozanimod enhances the thrombolytic effect of low-dose tPA, we measured the thrombus volume before and after ozanimod treatment by generating sequential z-stack images during in vivo 2-photon imaging (Fig. 5A-B). We found that ozanimod reduced thrombus volume (Fig. 5C), but did not affect lumen diameter (Fig. 5D), suggesting that S1PR1 modulation may enhance the thrombolytic effect of low-dose tPA by reducing thrombus volume.

As S1PR1 modulation is known to restrict the mobilization of peripheral lymphocytes, we examined whether ozanimod treatment could alter the composition of thrombus. To this end, we conducted immunostaining to examine the lymphocyte content within thrombus. Importantly, we found that ozanimod reduced the counts of $\mathrm{CD}^{+} \mathrm{T}$ cells and $\mathrm{CD} 45^{+}$cells within thrombus (Fig. 6A-D), suggesting that S1PR1 modulation could modify thrombus composition following thrombosis, which may involve its effect on the restriction of T cell mobilization.

\section{Discussion}

This 2-photon imaging study provides the novel evidence that selective S1PR1 modulation enhances the thrombolytic effect of low-dose tPA in cerebral arterioles after thrombosis. Based on real-time visualization and quantitation of blood flow along with thrombus volume in cortical arterioles, we found that ozanimod, an FDA-approved drug to treat multiple sclerosis, can reduce thrombus volume and improve flow velocity when combined with subthrombolytic dose of tPA. These findings are in line with our findings in a previous clinical trial showing that S1PR modulation improves collateral flow as an adjunctive therapy to tPA thrombolysis[9]. In addition, the antithrombotic activity of ozanimod and its effect to reduce the accumulation of $T$ cells within thrombus suggest that lymphocytes are among the major targets for the benefit of ozanimod to improve thrombolytic effect of low-dose tPA.

Low-dose tPA reduces the risk of hemorrhagic events but were not more effective as compared to the standard dose[3, 4]. Although the optimal low-dose tPA remains to be further explored, AIS patients with a high risk of symptomatic intracranial hemorrhage might benefit from low-dose tPA[3, 4]. In this respect, an adjunctive therapy is desirable to enhance the thrombolytic activity of low-dose tPA and maintain its safety advantage. The antithrombotic activity and effect to reduce T cells within thrombus place S1PR modulators as an ideal candidate for this purpose. Consistent with our recent study showing that a 
selective S1PR1 modulator RP101075, a metabolite of ozanimod, led to improved flow velocity following arteriole thrombosis induced by laser-irradiation[13], the present study has demonstrated that the addition of ozanimod to subthrombolytic dose of tPA results in a highly significant improvement of flow velocity at least until 90 min after treatment. Notably, ozanimod treatment reduced thrombus volume, suggesting that lymphocytes are a major target for the anti-thrombotic activity of ozanimod. This postulation is supported by our previous findings that the anti-thrombotic activity of S1PR1 modulator RP101075 was abolished in animals devoid of lymphocytes but not in animals receiving antibody depletion of monocytes and neutrophils[13].

Although tPA has been accepted as a standard of care for AIS patients, complete recanalization can only be achieved in less than one-third of patients receiving tPA thrombolysis[1, 18]. Reocclusion occurs in approximately one-third of patients following initial recanalization and accounts for two-thirds of deterioration after initial improvement $[1,18]$. These observations suggest that clinical deterioration after successful thrombolysis is related to continued vascular occlusive events. As small vessels such as arterioles and capillaries are particularly vulnerable to injury after cerebral ischemia, microvascular endothelial cells may be targeted by inflammatory cells that initiate thrombosis and prevent reflow following tPA thrombolysis[5, 19]. These considerations underscore the need for adjunctive therapies to enhance the benefit of thrombolysis. In the present study, we show that ozanimod can reduce the accumulation of T cells within the thrombus, suggesting the benefit of S1PR1 modulation to restrict inflammatory thrombosis and improve reflow following tPA thrombolysis.

The present results also provide strong support to our recent clinical trial showing that a S1PR modulator fingolimod enhances the efficacy of delayed tPA administration in AIS patients by promoting anterograde reperfusion and retrograde collateral flow (identifier: NCT02002390)[9]. In that randomized, blinded endpoint trial, we enrolled 46 patients with internal carotid artery or middle cerebral artery proximal occlusion within 4.5 to $6 \mathrm{~h}$ from onset[9]. The 23 patients receiving fingolimod plus tPA had better clinical improvement at $24 \mathrm{~h}$ and a favorable shift of $\mathrm{mRS}$ at day 90 . Importantly, these 23 patients receiving fingolimod plus tPA exhibited a reduction in the perfusion lesion and suppressed infarct growth. This is accompanied by improved anterograde reperfusion of downstream territory and retrograde reperfusion from collateral circulation[9]. Thus, these trial results suggest that S1PR modulation may enhance the efficacy of tPA thrombolysis. The present results lend further credence to such possibility. In this regard, larger clinical trials should be planned to assess the effects of S1PR modulators versus placebo in thrombolytic and nonthrombolytic cohorts. Results from this design may shed light on the postulation that S1PR modulation can enhance tPA thrombolysis.

In summary, our results demonstrate that the benefit of S1PR1 modulation enhance thrombolytic activity of low-dose tPA. These results will facilitate future design of larger clinical trials to determine the benefit of S1PR1 modulators as an adjunctive therapy to tPA thrombolysis.

\section{Abbreviations}


S1PR1: sphingosine-1-phosphate receptor 1; tPA: tissue plasminogen activator;

AIS: acute ischemic stroke.

\section{Declarations}

\section{Acknowledgements}

N/A

\section{Author contributions}

Q.L. formulated the study concept. H.L., Y.K., C.Q. and D.W. performed experiments. H.L., Y.K. and W.Z. analyzed the data, interpreted the results, and assisted preparation of the manuscript. Q.L. and H.L. wrote the paper.

\section{Funding}

This study was supported by Natural Science Foundation of Tianjin Education Commission(2020KJ148), Natural Science Foundation of Tianjin (18JCZDJC97600) and National Natural Science Foundation of China (82071327).

\section{Availability of data and materials}

All data generated during and/or analyzed during the current study are included in this published article. All source data supporting the findings of this manuscript are available from the corresponding authors upon request.

\section{Ethics approval and consent to participate}

N/A

\section{Consent for publication}

$\mathrm{N} / \mathrm{A}$

\section{Competing interests}

The authors report no competing interests

\section{References}

1. Thiebaut AM, Gauberti M, Ali C, Martinez De Lizarrondo S, Vivien D, Yepes M, Roussel BD. The role of plasminogen activators in stroke treatment: fibrinolysis and beyond. Lancet Neurol. 2018;17:112132. 
2. Anderson CS, Robinson T, Lindley RI, Arima H, Lavados PM, Lee TH, Broderick JP, Chen X, Chen G, Sharma VK, et al. Low-Dose versus Standard-Dose Intravenous Alteplase in Acute Ischemic Stroke. N Engl J Med. 2016;374:2313-23.

3. Wang X, Lee KJ, Moullaali TJ, Kim BJ, Li Q, Bae HJ, Carcel C, Delcourt C, Arima H, Sato S, et al. Who will benefit more from low-dose alteplase in acute ischemic stroke? Int J Stroke. 2020;15:39-45.

4. Wang X, Robinson TG, Lee TH, Li Q, Arima H, Bath PM, Billot L, Broderick J, Demchuk AM, Donnan G, et al. Low-Dose vs Standard-Dose Alteplase for Patients With Acute Ischemic Stroke: Secondary Analysis of the ENCHANTED Randomized Clinical Trial. JAMA Neurol. 2017;74:1328-35.

5. Fu Y, Liu Q, Anrather J, Shi FD. Immune interventions in stroke. Nat Rev Neurol. 2015;11:524-35.

6. Chamorro A, Dirnagl U, Urra X, Planas AM. Neuroprotection in acute stroke: targeting excitotoxicity, oxidative and nitrosative stress, and inflammation. Lancet Neurol. 2016;15:869-81.

7. Xue $\mathrm{M}$, Yong VW. Neuroinflammation in intracerebral haemorrhage: immunotherapies with potential for translation. Lancet Neurol. 2020;19:1023-32.

8. De Meyer SF, Denorme F, Langhauser F, Geuss E, Fluri F, Kleinschnitz C. Thromboinflammation in Stroke Brain Damage. Stroke. 2016;47:1165-72.

9. Tian DC, Shi K, Zhu Z, Yao J, Yang X, Su L, Zhang S, Zhang M, Gonzales RJ, Liu Q, et al. Fingolimod enhances the efficacy of delayed alteplase administration in acute ischemic stroke by promoting anterograde reperfusion and retrograde collateral flow. Ann Neurol. 2018;84:717-28.

10. Li YJ, Shi SX, Liu Q, Shi FD, Gonzales RJ. Targeted role for sphingosine-1-phosphate receptor 1 in cerebrovascular integrity and inflammation during acute ischemic stroke. Neurosci Lett. 2020;735:135160.

11. Iwasawa E, Ishibashi S, Suzuki M, Li F, Ichijo M, Miki K, Yokota T. Sphingosine-1-Phosphate Receptor 1 Activation Enhances Leptomeningeal Collateral Development and Improves Outcome after Stroke in Mice. J Stroke Cerebrovasc Dis. 2018;27:1237-51.

12. Brait VH, Tarrason G, Gavalda A, Godessart N, Planas AM. Selective Sphingosine 1-Phosphate Receptor 1 Agonist Is Protective Against Ischemia/Reperfusion in Mice. Stroke. 2016;47:3053-6.

13. Li H, Zhou X, Li Y, Ma X, Gonzales RJ, Qiu S, Shi FD, Liu Q. The selective sphingosine 1-phosphate receptor 1 modulator RP101075 improves microvascular circulation after cerebrovascular thrombosis. FASEB J. 2019;33:10935-41.

14. Nishimura N, Schaffer CB, Friedman B, Tsai PS, Lyden PD, Kleinfeld D. Targeted insult to subsurface cortical blood vessels using ultrashort laser pulses: three models of stroke. Nat Methods. 2006;3:99108.

15. Lou N, Takano T, Pei Y, Xavier AL, Goldman SA, Nedergaard M. Purinergic receptor P2RY12dependent microglial closure of the injured blood-brain barrier. Proc Natl Acad Sci U S A. 2016;113:1074-9.

16. Kleinfeld D, Mitra PP, Helmchen F, Denk W. Fluctuations and stimulus-induced changes in blood flow observed in individual capillaries in layers 2 through 4 of rat neocortex. Proc Natl Acad Sci U S A. 1998;95:15741-6. 
17. Sun N, Shen Y, Han W, Shi K, Wood K, Fu Y, Hao J, Liu Q, Sheth KN, Huang D, Shi FD. Selective Sphingosine-1-Phosphate Receptor 1 Modulation Attenuates Experimental Intracerebral Hemorrhage. Stroke. 2016;47:1899-906.

18. Alexandrov AV, Grotta JC. Arterial reocclusion in stroke patients treated with intravenous tissue plasminogen activator. Neurology. 2002;59:862-7.

19. Stoll G, Nieswandt B. Thrombo-inflammation in acute ischaemic stroke - implications for treatment. Nat Rev Neurol. 2019;15:473-81.

\section{Figures}

A

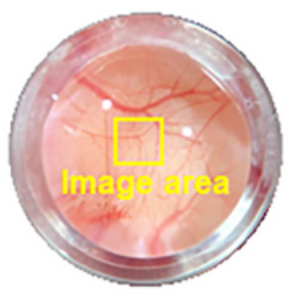

$\mathrm{B}$

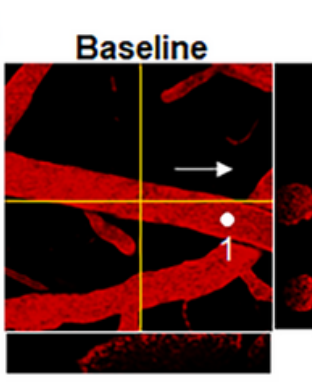

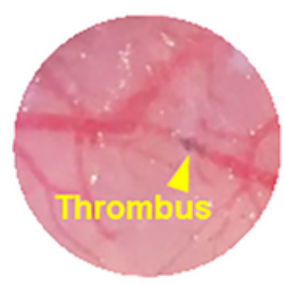

60 min after thrombosis

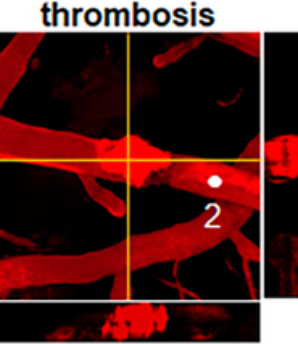

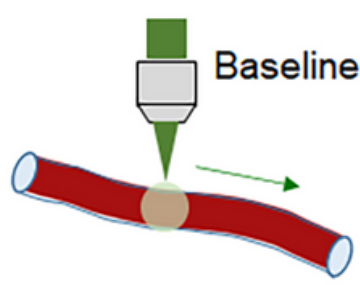

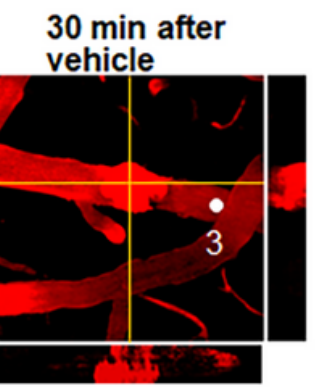

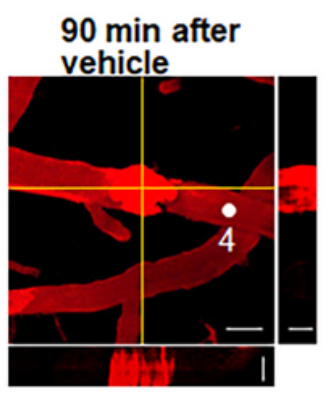

$60 \mathrm{~min}$ after thrombosis
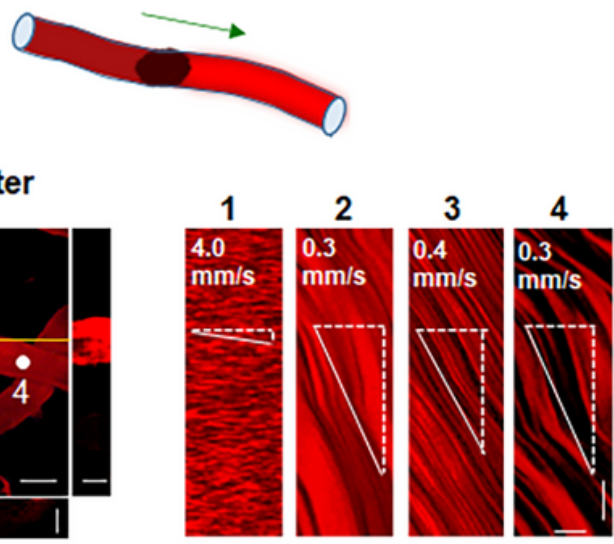

C

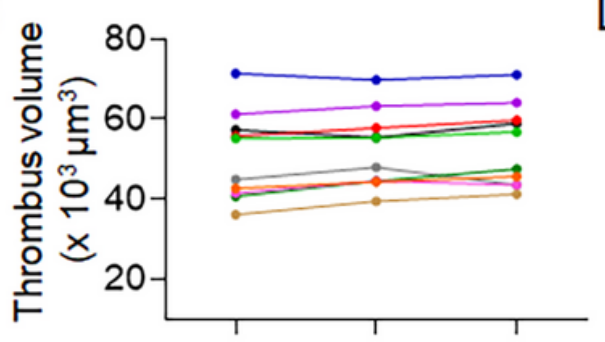

$\mathrm{D}$

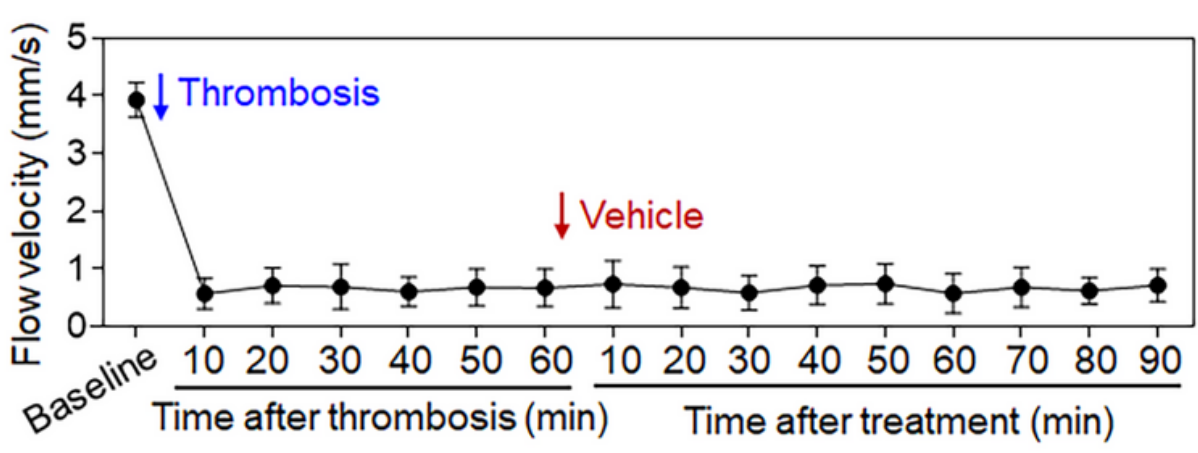

\section{Figure 1}

Effect of thrombosis on flow velocity in cortical arterioles measured by 2-photon laser scanning microscopy. A. Images and diagrams show the induction of thrombosis in cortical arterioles using 2photon laser scanning microscopy. B. Z-stack Images show cortical arterioles at baseline, 60 min after thrombosis and up to $90 \mathrm{~min}$ after vehicle treatment. 3D images show horizontal and vertical views of thrombus. The yellow lines indicate the horizontal and vertical cross sections of thrombus. White arrows indicate the direction of blood flow. White dots and numbers indicate individual location to measure flow 
velocity. Scale bar: $50 \mu \mathrm{m}$ in thrombus images, $30 \mu \mathrm{m}$ in cross sections. Line scans were performed to measure flow velocity along the longitudinal axis of arterioles of interest (flow velocity is expressed as $\mathrm{mm}$ per second). The slopes $(\Delta \mathrm{x} / \Delta \mathrm{t})$ of the measurement-angles are proportional to flow velocity. Scale bar: $40 \mu \mathrm{m}$. Time bar: $0.1 \mathrm{sec}$. C. Line graph shows thrombus volume at $60 \mathrm{~min}$ after thrombosis, $30 \mathrm{~min}$ and 90 min after vehicle treatment. D. Flow velocity at indicated time points. $n=10$ per group. Mean \pm SD.
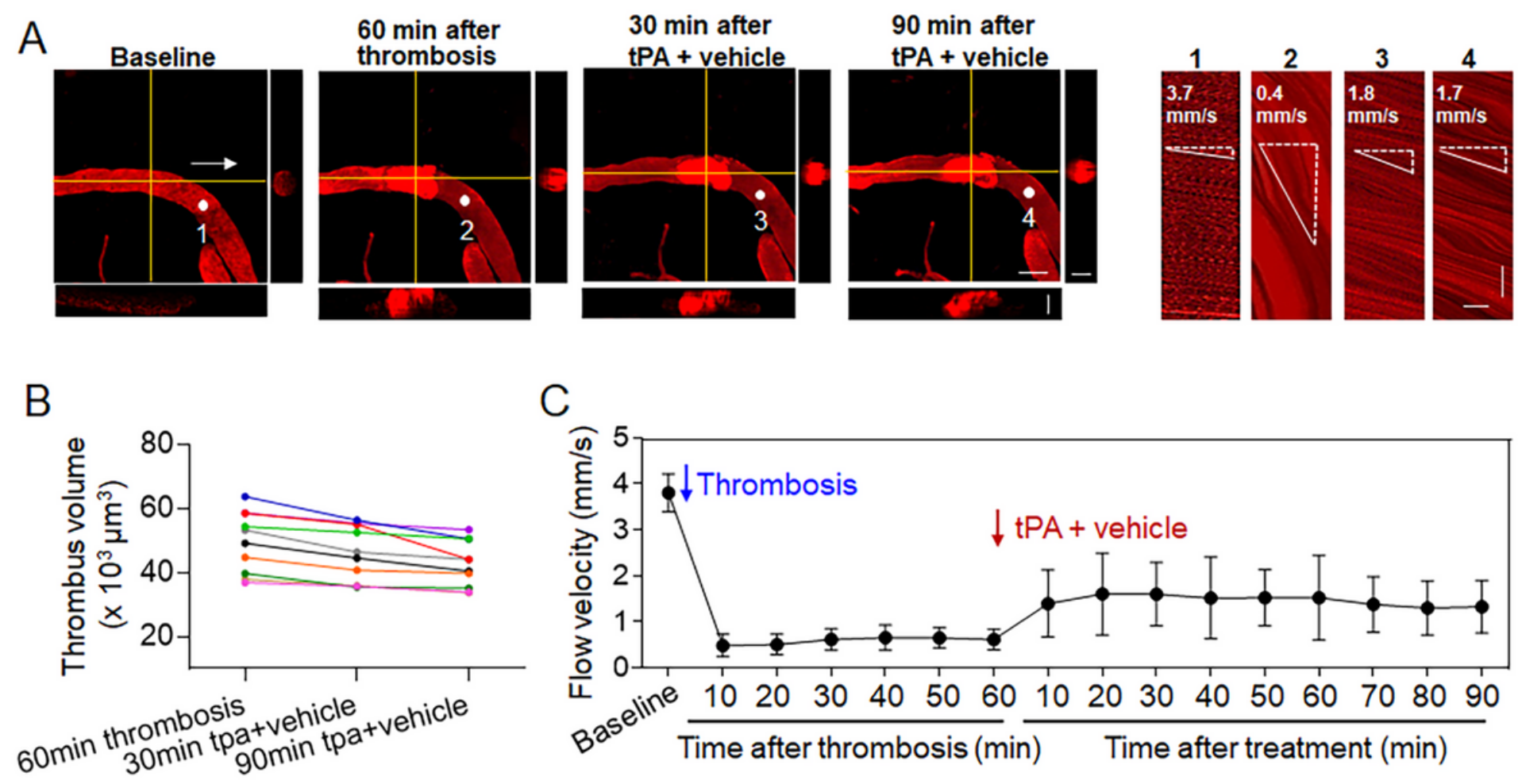

\section{Figure 2}

Effect of low-dose tPA on flow velocity in cortical arterioles following thrombosis. A. Z-stack Images show cortical arterioles and flow velocity at baseline, $60 \mathrm{~min}$ after thrombosis and up to $90 \mathrm{~min}$ after vehicle plus low-dose tPA ( $5 \mathrm{mg} / \mathrm{kg}$, i.v.) treatment. 3D images show horizontal and vertical views of thrombus. The yellow lines indicate the horizontal and vertical cross sections of thrombus. White arrows indicate the direction of blood flow. White dots and numbers indicate individual location to measure flow velocity. Scale bar: $50 \mu \mathrm{m}$ in thrombus images, $30 \mu \mathrm{m}$ in cross sections. Line scans were performed to measure flow velocity along the longitudinal axis of arterioles of interest (flow velocity is expressed as $\mathrm{mm}$ per second). The slopes $(\Delta x / \Delta t)$ of the measurement-angles are proportional to flow velocity. Scale bar: 40 $\mu \mathrm{m}$. Time bar: $0.1 \mathrm{sec}$. B. Line graph shows thrombus volume at $60 \mathrm{~min}$ after thrombosis, $30 \mathrm{~min}$ and 90 min after tPA + vehicle treatment. C. Flow velocity at indicated time points. $n=10$ per group. Mean \pm SD. 

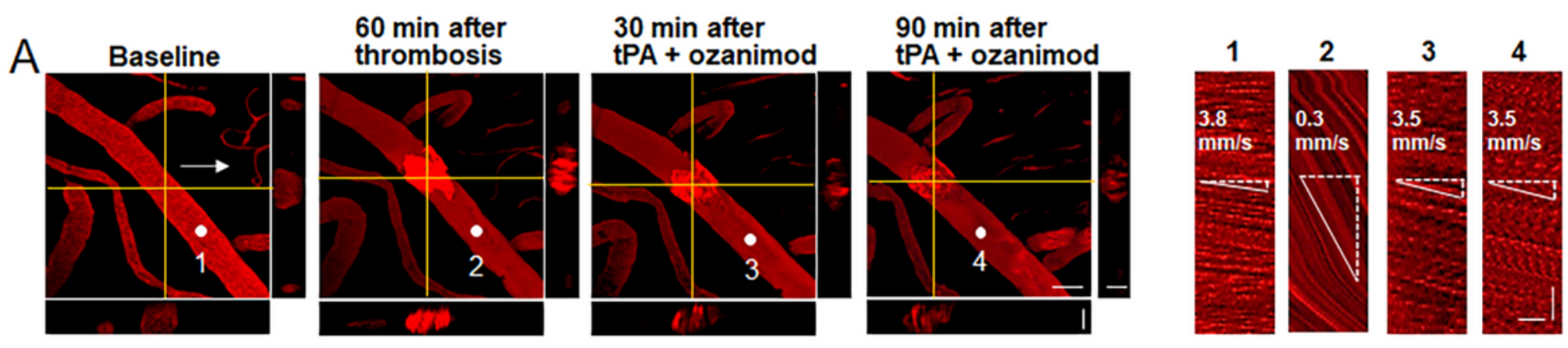

B

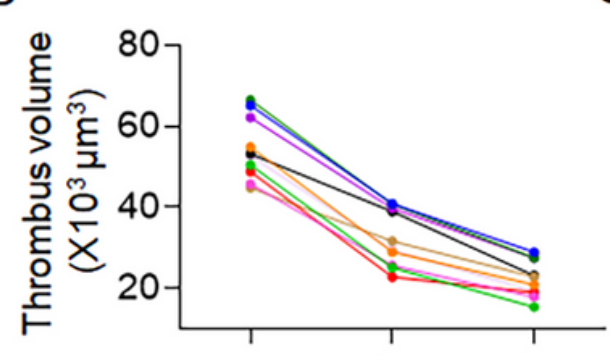

$60 \mathrm{~min}$ thrombosis
$30 \mathrm{~min}$ tpa+Ozimod
$90 \mathrm{~min}$ tpat

C

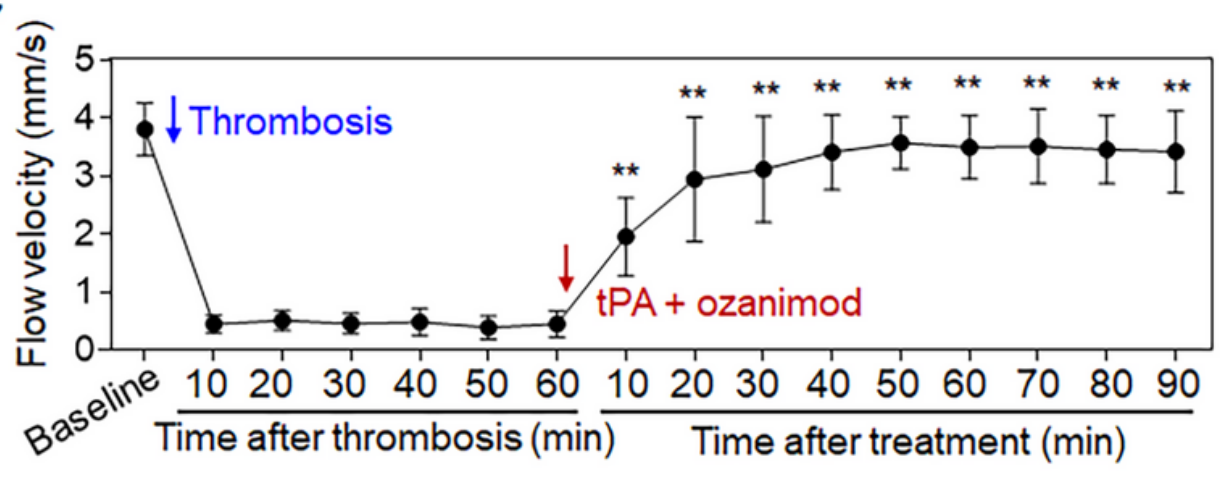

Figure 3

Effect of ozanimod and low-dose tPA treatment on flow velocity in cortical arterioles following thrombosis. A. Z-stack Images show cortical arterioles and flow velocity at baseline, 60 min after thrombosis and up to $90 \mathrm{~min}$ after ozanimod $(0.6 \mathrm{mg} / \mathrm{kg}$, i.v.) plus low-dose tPA ( $5 \mathrm{mg} / \mathrm{kg}$, i.v.) treatment. 3D images show horizontal and vertical views of thrombus. The yellow lines indicate the horizontal and vertical cross sections of thrombus. White arrows indicate the direction of blood flow. White dots and numbers indicate individual location to measure flow velocity. Scale bar: $50 \mu \mathrm{m}$ in thrombus images, 30 $\mu \mathrm{m}$ in cross sections. Line scans were performed to measure flow velocity along the longitudinal axis of arterioles of interest (flow velocity is expressed as mm per second). The slopes $(\Delta x / \Delta t)$ of the measurement-angles are proportional to flow velocity. Scale bar: $40 \mu \mathrm{m}$. Time bar: $0.1 \mathrm{sec}$. B. Line graph shows thrombus volume at $60 \mathrm{~min}$ after thrombosis, $30 \mathrm{~min}$ and $90 \mathrm{~min}$ after TPA + Ozanimod treatment. C. Flow velocity at indicated time points. $n=10$ per group. Mean $\pm S D$. ${ }^{*} p<0.01$ versus 60 min after thrombosis. ${ }^{*} \mathrm{p}<0.01$ versus vehicle $+\mathrm{tPA}$ group. 

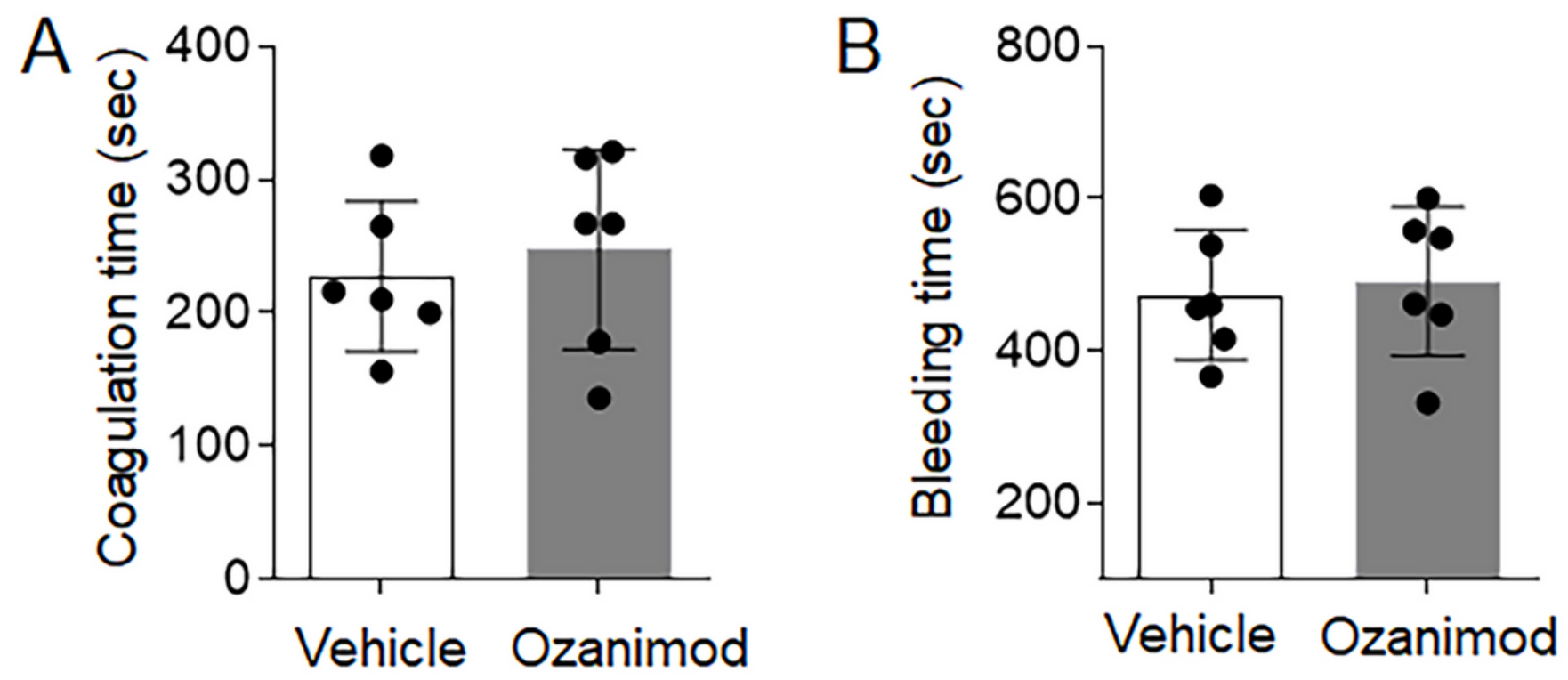

Figure 4

Effect of ozanimod on coagulation and bleeding time. A. Bar graph shows coagulation time in mice receiving vehicle or ozanimod $(0.6 \mathrm{mg} / \mathrm{kg}$, i.v. $)$. B. Bar graph shows bleeding time in mice receiving vehicle or ozanimod ( $0.6 \mathrm{mg} / \mathrm{kg}$, i.v.). $\mathrm{n}=6$ per group. Mean \pm SD. 
A
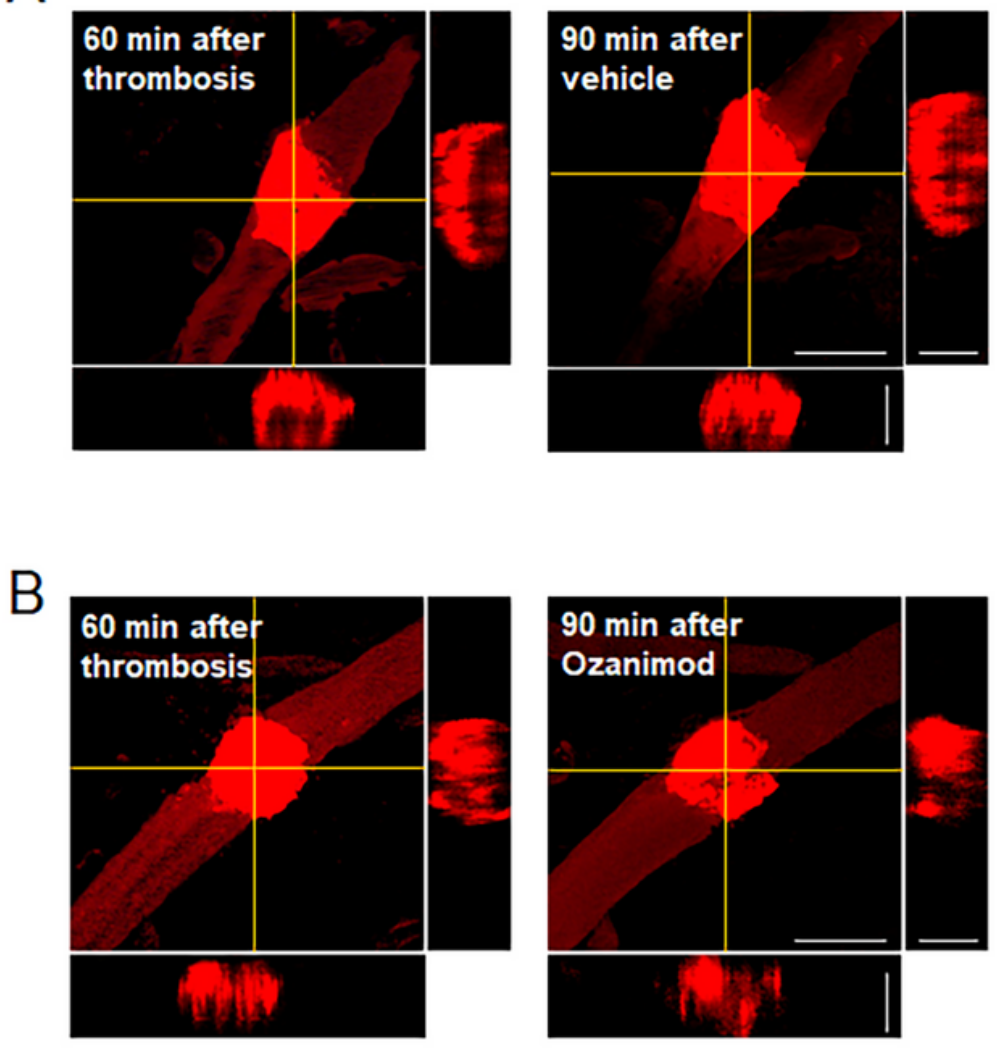

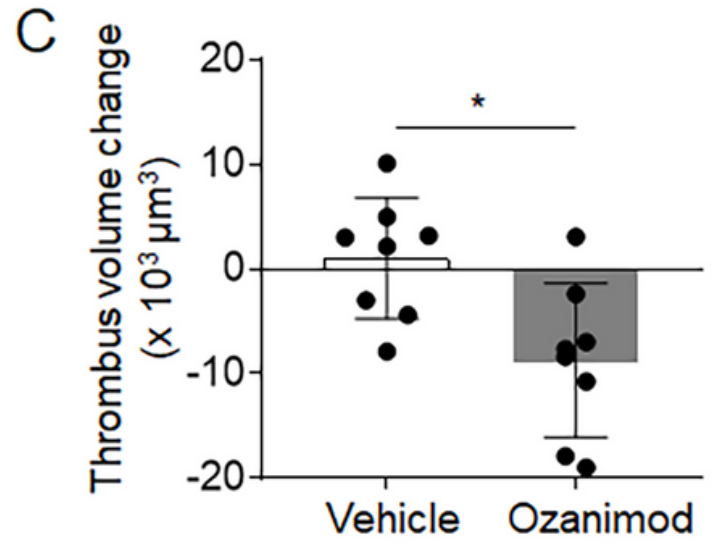

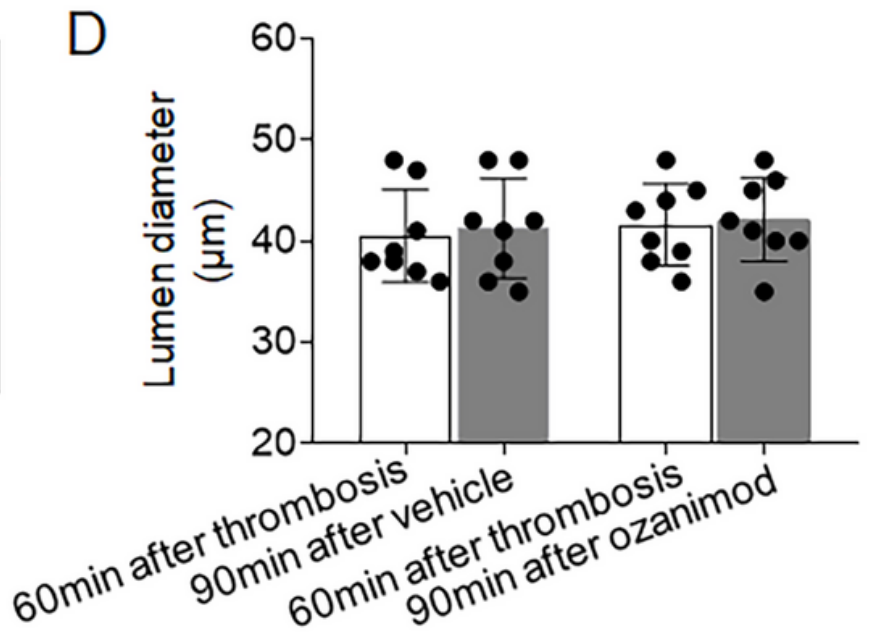

Figure 5

Effect of ozanimod on thrombus volume and lumen diameter in cortical arterioles following thrombosis. A-B. Z-stack Images show thrombus at $60 \mathrm{~min}$ after thrombosis and $90 \mathrm{~min}$ after ozanimod $(0.6 \mathrm{mg} / \mathrm{kg}$, i.v.) or vehicle treatment. 3D images show horizontal and vertical views of thrombus. The yellow lines indicate the horizontal and vertical cross sections of thrombus. Scale bar: $50 \mu \mathrm{m}$ in thrombus images, 30 $\mu \mathrm{m}$ in cross sections. C. Bar graph shows the changes of thrombus volume at $90 \mathrm{~min}$ after ozanimod or vehicle treatment. Volume change $=$ thrombus volume at $90 \mathrm{~min}$ after treatment - baseline volume at 30 min after thrombosis. $\mathrm{n}=8$ per group. D. Bar graph shows the changes of lumen diameter at $90 \mathrm{~min}$ after ozanimod or vehicle treatment. Lumen diameter was calculated at $60 \mathrm{~min}$ after thrombosis and $90 \mathrm{~min}$ after ozanimod or vehicle treatment. $n=8$ per group. Mean \pm SD. ${ }^{*} p<0.05$. 

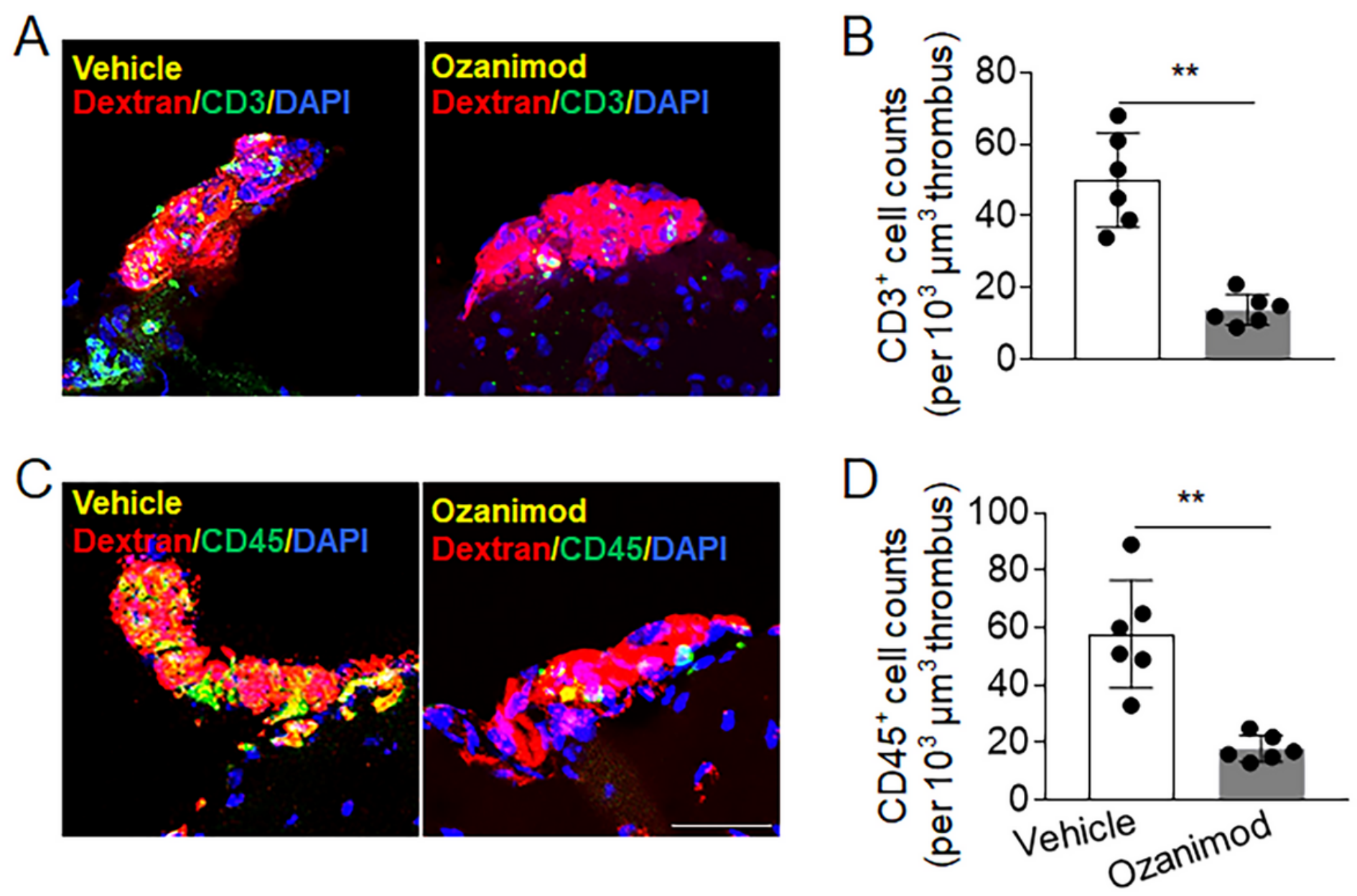

Figure 6

Effect of ozanimod on the composition of thrombus in cortical arterioles. Brain sections were prepared from brain tissues harvested at $90 \mathrm{~min}$ after ozanimod $(0.6 \mathrm{mg} / \mathrm{kg}$, i.v.) or vehicle treatment. A. Z-stack immunostaining images show CD3+ T cells (green) within Dextran Texas-Red labeled thrombus in cortical arterioles. B. Bar graph shows the counts of $\mathrm{CD} 3+\mathrm{T}$ cells within thrombus. C. Z-stack immunostaining images show CD 45+ cells (green) within Dextran Texas-Red labeled thrombus. D. Bar graph shows the counts of CD $45+$ cells (green) within thrombus. Scale bar: $40 \mu \mathrm{m} . \mathrm{n}=6$ per group. Mean \pm SD. ${ }^{*} \mathrm{p}<0.01$. 